\title{
Lanthanide Nitrates as Lewis Acids in the One-Pot Synthesis of 1,2,4-Oxadiazole Derivatives
}

\author{
Juliana A. Vale, ${ }^{*, a}$ Wagner M. Faustino, ${ }^{a}$ Davila de S. Zampieri, ${ }^{b}$ Paulo J. S. Moran, ${ }^{b}$ \\ José A. R. Rodrigues ${ }^{b}$ and Gilberto F. de Sá ${ }^{c}$ \\ ${ }^{a}$ Departamento de Química, Universidade Federal da Paraíba, 58059970 João Pessoa-PB, Brazil \\ ${ }^{b}$ Instituto de Química,Universidade Estadual de Campinas, CP 6154, \\ 13084-971Campinas-SP, Brazil \\ 'Departamento de Química Fundamental, CCEN, Universidade Federal de Pernambuco, \\ 50590470 Recife-PE, Brazil
}

\begin{abstract}
Neste trabalho relatamos o uso de nitratos de lantanídeos $\left[\mathrm{Ln}\left(\mathrm{NO}_{3}\right)_{3}\right]$ atuando como catalisador na síntese de uma única etapa de derivados de 3-benzoíla- e 3-acetila-1,2,4-oxadiazóis a partir de cetonas, nitrilas e ácido nítrico. Este é o primeiro exemplo da síntese em única etapa de preparação de derivados benzoíla e acetila-1,2,4-oxadiazóis, usando acetofenonas com grupos doadores de elétrons.
\end{abstract}

In this work we report the use of lanthanide nitrates $\left[\mathrm{Ln}\left(\mathrm{NO}_{3}\right)_{3}\right]$ acting as catalyst in direct onepot synthesis of 3-benzoyl- and 3-acetyl-1,2,4-oxadiazoles derivatives from ketones, nitriles and nitric acid. This is the first example of one-pot synthesis of benzoyl- and acetyl 1,2,4-oxadiazoles derivatives preparation using acetophenones derivates with electron-donator groups.

Keywords: lanthanide nitrates, Lewis acids, 1,2,4-oxadiazole derivates

\section{Introduction}

Lanthanide compounds have been extensively used as Lewis acids in the different reactions including asymmetric catalysis. The most important compounds include the use of chlorides, ${ }^{1}$ triflates, ${ }^{2}$ p-toluenesulphonates, ${ }^{3}$ dithiocarbamates complexes, ${ }^{4}$ alkoxides ${ }^{5}$ and others. ${ }^{6}$ On the other hand, the oxadiazole group is a key element of a number of biological activity such as ligands of benzodiazepine receptor, ${ }^{7}$ inhibidor of protein tyrosine phosphatase, ${ }^{8}$ agonists of muscarinic receptors, ${ }^{9}$ antiinflammatory agents, ${ }^{10}$ antiviral agents, ${ }^{11}$ cardiovascular ${ }^{12}$ and other medicinal activity. ${ }^{13}$ Also, 1,2,4-oxadiazole compounds have been applied as ligands of metal complexes ${ }^{14}$ and liquid crystalline materials. ${ }^{15,16}$

Synthesis of 1,2,4-oxadiazole derivatives by using ketones, nitriles and nitric acid as starting materials and $\mathrm{Fe}\left(\mathrm{NO}_{3}\right)_{3}$ as catalyst has been reported by Itoh et al. ${ }^{17}$ However, this method has the disadvantage of low reaction rates and drastic conditions, resulting in low reaction

*e-mail: julianadqf@yahoo.com.br yields. Recently, yttrium triflate $\left[\mathrm{Y}(\mathrm{OTf})_{3}\right]^{18}$ was applied instead of $\mathrm{Fe}\left(\mathrm{NO}_{3}\right)_{3}$ by using the same reaction condition in the synthesis of various 1,2,4-oxadiazole with low reaction times and good yields. Nonetheless, neither of these methodologies could be successfully applied in the synthesis of 1,2,4-oxadiazole contained electron-donating.

In the present work, we have proposed a simple methodology for the one-pot synthesis of 1,2,4-oxadiazole derivates, by using lanthanide nitrates prepared in situ as Lewis acids. We have investigated the rule of different ions on the reaction.

\section{Experimental}

General

All reagents were commercially available and used without any purification. ${ }^{1} \mathrm{H}$ and ${ }^{13} \mathrm{C}$ NMR spectra were recorded on a Varian $300-\mathrm{MHz}$ spectrometer. Mass spectrometer spectra were recorded on a Shimadzu GC-MS-QP 5000 gas chromatograph-mass spectrometer equipped with a $30 \mathrm{~m} \times 0.25 \mathrm{~mm} \times 0.25 \mu \mathrm{m}$ capillary 
column of fused silica, Supelco Simplicity $1^{\mathrm{TM}}$. All reactions were monitored by TLC. Flash column chromatography was carried out using 200-300 mesh silica gel at increased pressure.

Representative experimental procedure for synthesis of 3,5-substituted-1,2,4-oxadiazole compounds by use of lanthanide oxides

In a typical procedure, the lanthanide oxides $(0.3 \mathrm{mmol})$ and nitric acid $(0.3 \mathrm{mmol})$ were stirred at $50^{\circ} \mathrm{C}$ and then was added the ketone $(3 \mathrm{mmol})$. After $10 \mathrm{~min}$ the appropriated nitrile $(1 \mathrm{~mL})$ was added and the result mixture stirred at $80{ }^{\circ} \mathrm{C}$ for the appropriate time (Table 3). The reaction mixture was quenched with $\mathrm{NaHCO}_{3}$ and the product was repeatedly extracted with ethyl acetate. The combined organic phases were dried over anhydrous $\mathrm{MgSO}_{4}$, followed by evaporation of the solvent under reduced pressure. The purification of the residue was performed using flash column chromatography (silica gel, EtOAc-cyclohexane 10:90). All the products were identified by comparison of their spectral data and physical properties with authentic sample. The compounds $\mathbf{1}, \mathbf{2}, \mathbf{8}$ and $\mathbf{9}$ were characterized by comparison with spectral date reported in the literature date. ${ }^{17,18}$

\section{Characterization data for representative compounds}

3-(4-Bromobenzoyl)-5-phenyl-1,2,4-oxadiazole (3)

${ }^{1} \mathrm{H}$ NMR $\left(300 \mathrm{MHz}, \mathrm{CDCl}_{3}\right) \delta 8.26(\mathrm{dd}, 2 \mathrm{H}, J$ 7.5, $2.5 \mathrm{~Hz}), 8.24$ (dd, 2H, J 7.6, 2.5 Hz), 7.71 (d, 2H, J 8.5 Hz), $7.67(\mathrm{t}, 1 \mathrm{H}, J 7.0 \mathrm{~Hz}), 7.57$ (t, 2H, $J 7.5 \mathrm{~Hz}) .{ }^{13} \mathrm{C} \mathrm{NMR}$ $\left(75 \mathrm{MHz}, \mathrm{CDCl}_{3}\right) \delta 181.9,176.7,166.0,133.8,133.6$, 132.1, 132.1, 130.3, 129.3, 128.5, 123.2. MS (EI): $\mathrm{m} / z(\%)$ $329.9(17)\left[\mathrm{M}^{+}\right], 280.9(46), 206.9(100), 182.8(71), 154.8$ (34), 104.9 (71), 76.9 (37), 51.0 (17).

\section{3-Benzoyl-5-phenyl-1,2,4-oxadiazole (4)}

${ }^{1} \mathrm{H}$ NMR $\left(300 \mathrm{MHz}, \mathrm{CDCl}_{3}\right) \delta 8.34(\mathrm{~d}, 1 \mathrm{H}, J 8.5 \mathrm{~Hz})$, $8.27(\mathrm{~d}, 1 \mathrm{H}, J 8.5 \mathrm{~Hz}), 7.71(\mathrm{t}, 2 \mathrm{H}, J 7.5 \mathrm{~Hz}), 7.65(\mathrm{t}, 2 \mathrm{H}$, J 7.5), 7.51 (q, $2 \mathrm{H}, J 7.5 \mathrm{~Hz}) .{ }^{13} \mathrm{C} \mathrm{NMR}\left(75 \mathrm{MHz}, \mathrm{CDCl}_{3}\right.$ ) $\delta 123.4,128.5,128.7,129.2,130.7,133.4,134.6,135.1$, 166.2, 176.5, 183.1. MS (EI): $m / z$ (\%) 250.1 (3) [M+ $\left[\mathrm{M}^{+}, 145.1\right.$ (9), 105.1 (100), 77.0 (37), 51 (10).

\section{3-(4-Methylbenzoyl)-5-phenyl-1,2,4-oxadiazole (5)}

${ }^{1} \mathrm{H}$ NMR $\left(300 \mathrm{MHz}, \mathrm{CDCl}_{3}\right) \delta 8.26(\mathrm{dd}, 2 \mathrm{H}, J 8.5$, $1.5 \mathrm{~Hz}), 7.65$ (t, 1H, J 7.0 Hz), 7.59 ( t, 2H, J $8.0 \mathrm{~Hz}$ ), 7.36 (d, $2 \mathrm{H}, J 8.5 \mathrm{~Hz}), 2.46$ (s, 3H). ${ }^{13} \mathrm{C} \mathrm{NMR}\left(75 \mathrm{MHz}, \mathrm{CDCl}_{3}\right)$ $\delta$ 182.6, 176.4, 166.3, 145.9, 133.4, 132.7, 130.8, 129.5, 128.5, 123.4, 21.8. MS (EI): $\mathrm{m} / z$ (\%) $264.1(8)\left[\mathrm{M}^{+}\right], 239.1$
(1), 159.1 (5), 147.1 (4), 136.1 (2), 119.1 (100), 105.1 (51), 91.0 (27), 77.0 (23), 65.0 (10), 51.0 (6).

\section{3-(4-Methoxybenzoyl)-5-phenyl-1,2,4-oxadiazole (6)}

${ }^{1} \mathrm{H}$ NMR $\left(300 \mathrm{MHz}, \mathrm{CDCl}_{3}\right) \delta 8.36(\mathrm{dd}, 2 \mathrm{H}, J 6.5$ $\mathrm{Hz}), 7.64(\mathrm{t}, 1 \mathrm{H}, J 6.5 \mathrm{~Hz}), 7.58$ (t, $2 \mathrm{H}, J 6.5 \mathrm{~Hz}), 7.03(\mathrm{~d}$, $2 \mathrm{H}, J 7.0 \mathrm{~Hz}), 3.92(\mathrm{~s}, 3 \mathrm{H}) .{ }^{13} \mathrm{C} \mathrm{NMR}\left(75 \mathrm{MHz}, \mathrm{CDCl}_{3}\right)$ $\delta 181.3,176.3,166.4,164,9,133.3,133.3,129.2,128.5$, 128.2, 123.5, 114.1, 55.6. MS (EI): $m / z(\%) 280(22)\left[\mathrm{M}^{+}\right]$, 206.9 (4), 174.9 (12), 160.9 (5), 146.9 (10), 135.1 (100), 121.1 (3), 105.1 (54), 92 (10), 77.0 (44), 63.0 (9), 51.0 (11).

3-(4-Nitrobenzoyl)-5-phenyl-1,2,4-oxadiazole (7)

${ }^{1} \mathrm{H} \mathrm{NMR}\left(300 \mathrm{MHz}, \mathrm{CDCl}_{3}\right): \delta 8.55(\mathrm{~d}, 2 \mathrm{H}, J 7 \mathrm{~Hz}), 8.40$ $(\mathrm{d}, 2 \mathrm{H}, J 9 \mathrm{~Hz}), 8.27(\mathrm{~d}, 2 \mathrm{H}, J 7 \mathrm{~Hz}), 7.69(\mathrm{t}, 1 \mathrm{H}, J 7 \mathrm{~Hz})$, $7.60(\mathrm{t}, 2 \mathrm{H}, J 7.50 \mathrm{~Hz}) .{ }^{13} \mathrm{C} \mathrm{NMR}\left(75 \mathrm{MHz}, \mathrm{CDCl}_{3}\right)$ : $\delta$ 181.4, 177.1, 165.9, 151.1, 139.5, 133.8, 131.8, 129.4, 129.3, 128.61, 123.0. MS (EI): $\mathrm{m} / z$ (\%) 295.1 (17) $\left[\mathrm{M}^{+}\right]$, 149.9 (40), 120.1 (8) 105.1 (100), 92.0 (14), 77.0 (35), 76.0 (16), $51.0(10)$.

3-(4-Methylbenzoyl)-5-methyl-1,2,4-oxadiazole (10)

${ }^{1} \mathrm{H} \mathrm{NMR}\left(300 \mathrm{MHz}, \mathrm{CDCl}_{3}\right) \delta 8.14(\mathrm{~d}, 2 \mathrm{H}, J 6.5 \mathrm{~Hz})$, $7.34(\mathrm{~d}, 2 \mathrm{H}, J 8 \mathrm{~Hz}), 2.73$ (s, 3H), $2.46(\mathrm{~s}, 3 \mathrm{H}) .{ }^{13} \mathrm{C} \mathrm{NMR}$ $\left(75 \mathrm{MHz}, \mathrm{CDCl}_{3}\right): \delta 182.4,177.4,165.8,145.8,132.6$, 130.7, 129.5, 21.8, 12.3. MS (EI): $m / z(\%) 202.1(9)\left[\mathrm{M}^{+}\right]$, 177.1 (1), 160.1 (10), 145.1 (2), 132.1 (10), 119.1 (100), 104.1 (2), 91.0 (32), 77.0 (3), 65.0 (12), 51.0 (3).

\section{Results and Discussion}

In a preliminary screening, we carried out the reaction of benzonitrile, using europium nitrate prepared in situ from europium oxide or from europium dithiocarbamate with nitric acid and 4-chloroacetophenone at $80{ }^{\circ} \mathrm{C}$. The results are compared with those obtained from the europium triflate and were shown in Table 1.

All of the three europium compounds gave similar yields; however, the reactions with europium nitrate and europium ditiocarbamate were faster than those with europium triflate. The similarity between the results for the three Lewis acids indicates that same intermediate is formed in all of the three reactions. Probably the europium nitrate formed in situ acts as Lewis acid in the enolization of the ketones in all of the cases.

The lanthanide nitrates is a salt of easy preparation from available lanthanide oxides, cheaper and more stable than others salts and lanthanide compounds. We have prepared and applied on the reaction described above nitrates of different metals, in order to investigate their role upon the reaction. The results are summarized on Table 2. 
Table 1. Europium compounds catalyzed synthesis of 3-(4-chlorobenzoyl)-5-methyl-1,2,4-oxadiazole (1)

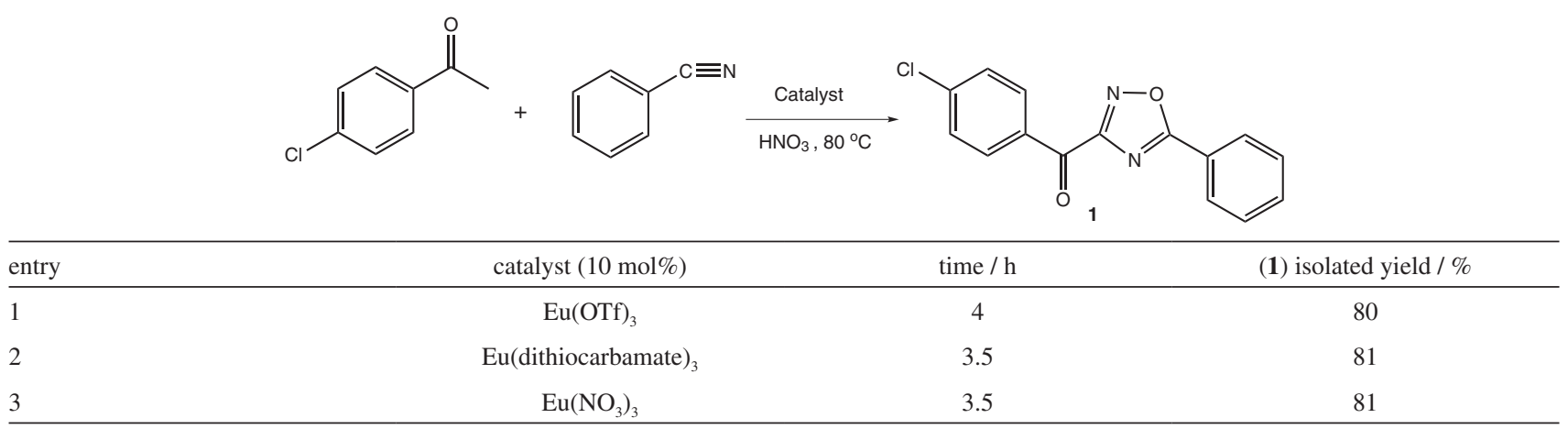

As it can be seen on Table 2, the use of $10 \mathrm{~mol} \%$ of lanthanide catalyst (entry 1-6) promoted the reaction between 4-chloroacetophenone and benzonitrile in the presence of nitric acid to give 3-(4-chlorobenzoyl)-5phenyl-1,2,4-oxadiazole (1) in good yield. Ytterbium

Table 2. Lanthanide nitrate catalyzed synthesis of 3-(4-chlorobenzoyl)5-methyl-1,2,4-oxadiazole (1)

\begin{tabular}{lccc}
\hline entry & $\begin{array}{c}\text { catalyst } \\
(10 \mathrm{~mol} \%)\end{array}$ & time / $\mathrm{h}$ & $\begin{array}{c}\text { (1) isolated } \\
\text { yield / \% }\end{array}$ \\
\hline 1 & $\mathrm{La}\left(\mathrm{NO}_{3}\right)_{3}$ & 4 & 70 \\
2 & $\mathrm{Nd}\left(\mathrm{NO}_{3}\right)_{3}$ & 3.5 & 72 \\
3 & $\mathrm{Eu}\left(\mathrm{NO}_{3}\right)_{3}$ & 3.5 & 81 \\
4 & $\mathrm{Gd}\left(\mathrm{NO}_{3}\right)_{3}$ & 3 & 81 \\
5 & $\mathrm{Er}\left(\mathrm{NO}_{3}\right)_{3}$ & 1.5 & 83 \\
6 & $\mathrm{Yb}\left(\mathrm{NO}_{3}\right)_{3}$ & 1.5 & 86 \\
\hline
\end{tabular}

catalyst proved to be the most effective (Table 2, entry 6). The product was obtained in $86 \%$ in just $1.5 \mathrm{~h}$ at $80^{\circ} \mathrm{C}$. The quantity of nitric acid was controlled to avoid the formation of benzoic acid from hydrolysis of benzonitrile.

Due to the excellent result obtained with $\mathrm{Yb}\left(\mathrm{NO}_{3}\right)_{3}$, it was applied for the synthesis of several 1,2,4-oxadiazole derivatives contained electron-withdrawing and electrondonating substituents in the aromatic ring of ketones. The results are given in the Table 3.

As it was shown in Table 3, a large variety of ketones can be converted into the corresponding 1,2,4-oxadiazole compounds with good to excellent yields. All yields with $\mathrm{Yb}\left(\mathrm{NO}_{3}\right)_{3}$ were higher than those obtained using $\mathrm{Fe}\left(\mathrm{NO}_{3}\right)_{3}$ as catalyst and short time reaction than those obtained by use $\mathrm{Y}(\mathrm{OTf})_{3} \cdot{ }^{18}$ The reactivity of benzonitrile was higher than the acetonitrile in terms of reaction time and isolated yield (compared compounds $(\mathbf{1}, \mathbf{8}),(\mathbf{4}, \mathbf{9})$ and $(\mathbf{5}, \mathbf{1 0})$ ).

Table 3. Yterbium nitrate (III) catalyzed synthesis of 1,2,4-oxadiazole derivatives<smiles></smiles>

\begin{tabular}{lccccc}
\hline entry & \multicolumn{5}{c}{ 2-10 } \\
\hline 1 & $\mathrm{R}^{1}$ & $\mathrm{R}^{2}$ & time / h & compounds & isolated yield / \% \\
2 & $\mathrm{~F}$ & $\mathrm{C}_{6} \mathrm{H}_{5}$ & 1 & $\mathbf{2}$ & 87 \\
3 & $\mathrm{Br}$ & $\mathrm{C}_{6} \mathrm{H}_{5}$ & 1 & $\mathbf{3}$ & 83 \\
4 & $\mathrm{H}$ & $\mathrm{C}_{6} \mathrm{H}_{5}$ & 1.5 & $\mathbf{4}$ & $\mathbf{5}$ \\
5 & $\mathrm{CH}_{3}$ & $\mathrm{C}_{6} \mathrm{H}_{5}$ & 3 & $\mathbf{5}$ & 73 \\
6 & $\mathrm{OCH}_{3}$ & $\mathrm{C}_{6} \mathrm{H}_{5}$ & 3 & $\mathbf{6}$ & 54 \\
7 & $\mathrm{NO}_{2}$ & $\mathrm{C}_{6} \mathrm{H}_{5}$ & 3 & $\mathbf{8}$ & 65 \\
8 & $\mathrm{Cl}$ & $\mathrm{CH}_{3}$ & 2 & $\mathbf{9}$ & 50 \\
9 & $\mathrm{H}$ & $\mathrm{CH}_{3}$ & 5 & $\mathbf{1 0}$ & 45 \\
\hline
\end{tabular}


Furthermore, the reaction occurred with ketones with electron-withdrawing and electron-donating at aromatic ring (Table 3, entries 4, 5 and 9) and with unsubstituted ring (Table 3, entries 3 and 8). This is the first time that 3-benzoyl- and 3-acetyl-1,2,4-oxadiazole derivates with electron donor substituent is synthesized one-pot.

\section{Conclusions}

In conclusion, we have demonstrated the effectiveness of the use of lanthanide nitrates as catalyst in the one-pot synthesis of 3-acetyl- and 3-benzoyl-1,2,4-oxadiazole derivates in good yield and short reaction times, when compared with other reported methodologies. Additionally, the reaction was efficient for aromatic ketones substituted with electron-donating and electron-withdrawing groups in the aromatic ring.

\section{Acknowledgments}

The authors thank the PRONEX-FACEPE-CNPq (APQ-0859-1.06/08) for the financial support.

\section{Supplementary Information}

Spectra of synthesized compounds are available free of charge at http://jbcs.sbq.org.br as PDF file.

\section{References}

1. Aspinal, H. C.; Bickley J. F.; Greeves, N.; Kelly, R. V.; Organometallics 2005, 24, 3458.

2. Li, C. J.; Chen, L.; Chem. Soc. Rev. 2006, 35, 68; Kobayashi, S.; Chem. Rev. 2002, 102, 2227.

3. Parac-Vogt, T. N.; Delersnyder, K.; Mehdi, H.; Horvath, I.; Binnemans, K.; Tetrahedron 2007, 63, 9063.

4. Vale, J. A; Faustino, W. M.; Menezes, P. H.; de Sá, G. F.; Chem. Commun. 2006, 31, 3340; Vale, J. A; Faustino, W. M.; Menezes, P. H.; de Sá, G. F.; J. Braz. Chem. Soc. 2006, 17, 829; Faustino, W. M.; Malta, O. L.; de Sá, G. F.; J. Phys. Chem. A 2006, 110, 2510 .

5. Johnson, J. S.; Greszler, S. N.; Organic Lett. 2009, 11, 827.

6. Inanaga, J.; Furono, H.; Hayano, T.; Chem. Rev. 2002, 102, 2211.

7. Ahn, J.-M.; Boyle, N. A.; MacDonald, M. T.; Janda, K. D.; Mini Rev. Med.Chem. 2002, 2,463; Watjen, F.; Baker, R.; Engelstoff, M.; Herbert, R.; MacLeod, A.; Knight, A.; Merchant, K.; Moseley, J.; Saunders, J.; Swain, C. J.; Wong, E.; Springer, J. P.; J. Med. Chem. 1989, 32, 2282.
8. Amarasinghe, K. K. D.; Evidokimov, A. G.; Xu, K.; Clark, C. M.; Maier, M. B.; Srivastava, A.; Colson, A.-O.; Gerwe, G. S.; Stake, G. E.; Howard, B. W.; Pokross, M. E.; Gray, J. L.; Peters, K. G.; Bioorg. Med. Chem. Lett. 2006, 16, 4252.

9. Orlek, B. S.; Blaney, F. E.; Brown, F.; Clark, M. S. G.; Hadley, M. S.; Hatcher, J.; Riley, G. J.; Rosenberg, H. E.; Wadsworth, H. J.; Wyman, P.; J. Med. Chem. 1991, 34, 2726; Street, L. J.; Baker, R.; Book, T.; Kneen, C. O.; MacLeod, A. M.; Merchant, K. J.; Showell, G. A.; Saunders, J.; Herbert, R. H.; Freedman, S. B.; Harley, E. A.; J. Med. Chem. 1990, 33, 2690.

10. Leite, A. C. L.; Vieira, R. F.; de Faria, A. R.; Wanderley, A. G.; Afiatpour, P.; Ximenes, E. C. P. A.; Srivastava, R. M.; De Oliveira, C. F.; Medeiros, M. V.; Antunes, E.; Brondani, D. J.; Il Fármaco 2000, 55, 719; Nicolaides, D. N.; Fylaktakidou, K. C.; Litinas, K. E.; Hadjipavlou-Litina, D.; Eur. J. Med. Chem. 1998, 33, 715.

11. Diana, G. D.; Volkots, D. L.; Nitz, T. J.; Bailey, T. R.; Long, M. A.; Vescio, N.; Aldous, S.; Pevear, D. C.; Dutko, F. J.; J. Med. Chem. 1994, 37, 2421. Diana, G. D.; Rudewicz, P.; Pevear, D. C.; J. Med. Chem. 1995, 38, 1355.

12. Mashkovski, M. D.; Sokolov, S. D.; Vinogradova, S. M.; Yuzhakov, S. D.; Yermakova, V. N.; Drugs Future 1997, 22, 499.

13. Walker, C.; Boer, C.; Mazzoni, L.; Bacher, E.; Keller, T. H.; Joachim, F. W. D.; Bray-French, K.; Müller, T.; Bioorg. Med. Chem. Lett. 1998, 8, 3229; Keller, T. H.; Chem. Pharm. Bull. 2001, 49, 1009; Graul, A.; Tracy, M.; Castañer, J.; Drugs Future 1997, 22, 639; Naismith, R. W.; Frierson III, M. R.; Wong, S.; Lee, S. J.; Drugs Future 1992, 17, 871; Ono, M.; Haratake, M.; Saji, H.; Nakayama, M.; Bioorg. Med. Chem. Lett. 2008, 16, 6867; Dolenc, M. S.; Ziga, J.; Roskar, R.; Tetrahedron Lett. 2007, 48, 1465.

14. Steel, P. J.; Richardson, C.; Inorg. Chem. Commun. 2007, $10,884$.

15. Gallardo, H.; Cristiano, R.; Vieira, A. A.; Neves Filho, R. A. W.; Srivastava, R. M.; Synthesis 2008, 605; Parra, M. L.; Hidalgo, P. I.; Elgueta, E. Y.; Liq. Cryst. 2008, 35, 823.

16. de Freitas, J. J. R.; de Freitas J. C. R.; da Silva, L. P.; de Freitas Filho, J. R.; Kimura, G.Y.V.; Srivastava, R. M.; Tetrahedron Lett. 2007, 48, 6195; Outirite, M.; Lebrini, M.; Lagrenée, M.; Bentiss, F. J.; Heterocycl Chem. 2007, 44, 1529; Kaboudin, B; Saadati, F.; Tetrahedron Lett. 2007, 48, 2829.

17. Itoh, K.; Sakamaki, H.; Horiuchi, C.; Synthesis 2005, 12, 1935

18. Chuanming, Y.; Min, L.; Weike, S.; Yuanyuan, X.; Synth. Commun. 2007, 37, 4439; Ling, R.; Yoshida, M.; Mariano, P. S.; J. Org. Chem. 1996, 61, 4439.

Submitted: December 20, 2011

Published online: July 5, 2012

FAPESP has sponsored the publication of this article. 